\title{
Self-Corrective Autonomous Systems using Optimization Processes for Detection and Correction of Unexpected Error Conditions
}

\author{
Nicoladie Tam \\ Department of Biological Sciences, University of North Texas, USA
}

\begin{tabular}{|c|c|}
\hline Article Info & ABSTRACT \\
\hline Article history: & $\begin{array}{l}\text { A theoretical framework for autonomous self-detection and self-correction of } \\
\text { unexpected error conditions is derived by incorporating the principles of }\end{array}$ \\
\hline Received Sep 25, 2016 & operation in autonomous control in biological evolution. Using the \\
\hline Revised Nov 2, 2016 & biologically inspired principles, the time-dependent multi-dimensional \\
\hline Accepted Nov 17, 2016 & $\begin{array}{l}\text { disparity vector is used as a quantitative metric for detecting unexpected and } \\
\text { unforeseeable error conditions without any external assistance. The disparity }\end{array}$ \\
\hline Keyword: & $\begin{array}{l}\text { vector is a measure of the discrepancy between the expected outcome } \\
\text { predicted by the autonomous system and the actual outcome in the real } \\
\text { world. It is used as a measure to detect any unexpected or unforeseeable }\end{array}$ \\
\hline Autonomous systems & errors. The process for autonomous self-correction of the self-discovered \\
\hline Emotional processing & errors is an optimization process to minimize the errors represented by the \\
\hline Error correction & disparity vectors. The strategies for prioritizing the urgency of corrective \\
\hline Error detection & actions are also provided in the theoretical derivations. The criteria for any \\
\hline & $\begin{array}{l}\text { The criteria for the detection of the minimization and maximization of errors } \\
\text { are also provided in the autonomous optimization process. The biological } \\
\text { correspondences of the emotional responses in relation to the autonomic self- } \\
\text { corrective feedback systems are also provided. }\end{array}$ \\
\hline
\end{tabular}

Copyright $@ 2016$ Institute of Advanced Engineering and Science. All rights reserved.

Corresponding Author:

Nicoladie Tam,

Department of Biological Sciences, University of North Texas, Denton, TX 76023 USA.

Email: nicoladie.tam@unt.edu

\section{INTRODUCTION}

One of the most crucial problems in autonomous control is the ability to detect and correct unexpected errors autonomously without any external assistance. This intrinsic problem usually requires selfidentification of error conditions and self-correction of the identified error conditions autonomously [1]. Autonomous correction of self-discovered error is one of the most challenging problems to solve in autonomous control of any complex systems. These complex autonomous systems include, but not limited to, autonomous robots, autonomous swamp robots and biological organisms. Even though there is a great diversity of autonomous systems ranging from autonomous robots to living organisms, the computational problems in self-correction of error conditions faced with these systems are similar. That is, regardless of whether it is an inanimate robot, a living animal or human, the probability of survival is dependent on the ability to self-correct any internal error conditions that it encountered. Therefore, it is important to design a system that is capable of self-discovery of errors and self-correction of any error conditions, especially those unforeseen internal error conditions that are not embedded in the design of the system. 


\subsection{Feedback Control System in Robust Engineering Design for Error Correction}

One of the design principles in robust engineering design is the use of feedback systems in the control of autonomous systems. Feedback control is one of the standard modes of operation in order to correct for error conditions in engineering (cf. [2-5]). Sometimes, feedforward control is often used to improve the efficiency in providing fast response time [6]. Yet, these feedforward or feedback systems are embedded into the system by design with a priori knowledge of how the error conditions may exist. If the specific expected errors were known, it is easy to implement a feedback control system to correct for such error conditions. For instance, to prevent skidding in cars, feedback control system can be designed in the anti-lock brake system, because the physics of the error conditions for skidding in wet roads are known. However, if the error conditions are unknown or unexpected (such as software bugs caused by the malfunction of the control system [7]), then the corrective actions may not be designed in the system without the $a$ priori knowledge of how the system fails. In computer systems, one of the most common methods for identifying unexpected error in integrity of data is the use of checksum [8,9] or result-checking [10, 11]. Yet, these methods often assume the errors are external errors rather than internal errors. That is, if the system is a faulty system with design flaws internally (such as software programming bugs), then the ability to detect and self-correct internal fault is a daunting problem for designing a fully autonomous system.

\subsection{Engineering Design Problem for Unexpected Error Conditions}

Specifically, if the feedback control system itself is faulty, then the problem to self-correct the faulty condition within the control system itself is a much harder problem to solve. This is a crucial problem when the fault condition is undetermined beyond the original design of the system. Therefore, in order to design a truly autonomous system, it should be capable of self-correcting any unexpected error conditions that it discovers without any external guidance or external control. These unforeseeable error conditions may include the design flaws of the original implementation (which is often unpredictable when the system is operating in the real world).

\subsection{The Problem of Self-Discovery and Self-Correction of Internal Design Flaws}

Most traditional engineering designs assume that there is no design flaw, and the system should behave as expected by design. Yet, in the real world implementation, there are always unexpected and unforeseeable errors encountered in real life that exhibit design flaws in the system. For instance, the most common design flaws in software engineering are the existence of software bugs in the software system. Software bugs are the unforeseeable error conditions encountered in the real world implement of software applications that are beyond the expectation of the original design. That is, if the software implementation were operating as expected by the design, then there should not be any software bugs in the final product. However, in reality, every software engineer designer knows that software bugs are likely to exist in any implementation of the complex software system. Thus, the problem of self-discovering software bugs and self-correction of these bugs when they were discovered are a daunting problem for advanced autonomous intelligent software designs.

\subsection{Autonomous System with Self-Discovery and Self-Correction of Unexpected Errors}

One of the most challenging problems in engineering designs is the ability to design a system with the capability to self-discover internal errors, and then self-correct such errors once they were identified. The most difficult part of the problem for a truly autonomous system design is that ability to discover unforeseeable errors that are beyond the original designs. That is, the autonomous system should be able to correct any errors that it encounters, including the unexpected, unforeseeable design flaws, if it is truly autonomous.

The ability to self-correct the system's own fault conditions depends on the ability to identify error conditions had existed; otherwise, it would not be able to correct it. In most engineering designs, the final implementation always assume the product will operate as expected according to design. However, this assumption may not be valid in the real world because if there were design flaws, how would the machine know itself is erroneous?

\subsection{Detection of Unexpected Fault Conditions}

Most often, the design flaws are discovered and detected by some other external sources other than the implemented product itself. That is, if the autonomous product is a robot or a software program, it would rarely discover its own bug - the bugs are often discovered by some external source, such as another human. On the other hand, if the autonomous being is a living organism (such as human or animal), the organism itself often does not know its intrinsic fault conditions - those flaws are often discovered by some other humans. 
This paper is focused on addressing how to design autonomous systems that can self-discover unforeseeable fault conditions; not just foreseeable errors in interacting with the external world, but also unforeseeable internal faults and bugs conditions. It is addressing the next-generation design of autonomous systems that are capable of self-discover unforeseeable error conditions.

\subsection{Correction of Self-Discovered Error Conditions}

In the design of the next-generation autonomous self-corrective systems, not only do they need to discover unexpected error conditions, but also correct the self-discovered error conditions without any external help. That is, it requires a two-step process in error-recovery. The first step is to discover and identify the source of error conditions without any external assistance. The second step is to correct the error conditions and recover from the fault condition autonomously without any external guidance. For instance, an autonomous software system could be able to discover its own bugs and correct the bugs in the software code without any external assistance. Similarly, in a living organism, such as human, it would be able to discover and identify its own flaws, then correct its faults without getting help from someone else (i.e., without counseling by another person).

\section{METHODS}

Towards the goal of designing a self-corrective autonomous system, the first task is to detect the unexpected error conditions. The second task is to correct such discovered the error condition that it encounters. In order to discover any unexpected/unforeseeable error without external guidance, we implement one of the biologically inspired methods to discover and correct the unexpected errors autonomously. Since biological organisms are autonomous by nature, most of the principles of autonomous operation have often already been discovered in evolution. In biological animal systems, especially in higheranimals, including humans, the evolution of emotional responses is one of the intrinsic self-corrective principles discovered in nature to identify unexpected error conditions and recover from them [12, 13].

\subsection{Biologically Inspired Autonomous Error Recovery Process}

According to this theory of emotional processing, emotion is evolved as an internal feedback system that identifies error conditions, which is expressed as unhappy emotions [14]. The error recovery process is essentially the internal emotional resolution process to reach the contented state of happiness, which signifies the congruent state between the expected outcome and the actual outcome in the real world [15]. It is essentially an optimization process to minimize the disparity between the expected and the actual outcomes so that when the congruency between the expected and actual outcomes is reached, it is indicated by the state of happiness internally. The use of minimization [16] as the first and second order self-correcting method had been explored theoretically in other physical systems.

In other words, the error condition is self-discovered autonomously by the disparity between the expected and the actual outcomes, which is expressed as the unhappy emotional state. That is, the unhappy emotion is an internal feedback that indicates some error conditions have occurred that need to be fixed. This provides the autonomous process in error recovery, using emotion as an internal feedback to guide the error correction process to minimize the errors that it discovered.

Therefore, one of the methodologies to identify the existence of error conditions is to compare the difference between the predicted outcomes of the system and the actual outcomes in reality. This paper will derive a set of optimization process in autonomous recovery of errors based on the principles of operation inspired by the biological emotional resolution process.

\subsection{Self-Discovery of Unexpected Error Conditions}

Based on the principle of survival, the survivability of a system is dependent on the ability to predict the outcomes produced by the system are as accurately as possible. That is, the outcomes produced by the system should correspond to the actual outcomes in the real world environment. If there are discrepancies between the predicted/expected outcomes and the actuality in the real world, then the system may not survive as well as a system that can produce the outcome accurately. Therefore, the survivability of the system is dependent on the accuracy of producing outcomes that correspond to the real world environment. The higher the degree of congruency between the predicted and the actual outcomes, the greater the survivability of the system will be. Survivability is defined as the probability of survival for an autonomous system to operate and to function in the real world without catastrophic failure. 


\subsection{Principle of Survivability for Optimizing the Autonomous System}

Using this survivability principle, the goal of the design of the autonomous system is to increase its chance of survival. In order words, any fault conditions would decrease its chance of survival as a system. Thus, in designing a truly autonomous system, the problem to solve is to use the survivability of the system as a metric for optimization. In other words, the goal is to optimize the survival of the system as a whole in its operating principles, so that the system can discover unexpected errors without reliance on any external assistance. It will be shown below that biological autonomous systems, such as higher-animals and humans, use emotion as a feedback system to self-discover and self-correct any unexpected error conditions, even though those error conditions are unforeseen and unpredictable, without any a priori knowledge of the reality in the real world.

\subsection{Discrepancy Measure as a Metric to Self-Discover Error Conditions}

In order to discover unexpected errors without external guidance, one of the methodologies is to use the discrepancy between the predicted outcomes of the system and the actual outcomes in the real world as a measure of the existence of any error condition. The difference between these two outcomes would indicate some errors must have existed that caused the system to produce the inaccurate outcome. Thus, using this disparity measure, the system is able to self-discover any unexpected or unforeseen error conditions, without relying on any external guidance. It will be derived below that this disparity measure also corresponds to the unhappy emotion in animals as an autonomic response to the detection of error conditions generated internally by the animal as a feedback to correct the unexpected error conditions.

\section{SELF-DISCOVERY OF UNEXPECTED/UNFORESEEABLE ERROR CONDITIONS}

Let $\mathbf{u}(t)$ be the time-dependent multi-dimensional vector representing the predicted outcome produced by the autonomous system, and $\hat{\mathbf{u}}(t)$ the time-dependent multi-dimensional vector representing actual outcome in the real world, then the time-dependent disparity, $\mathbf{v}(t)$, between the expected and the actual outcomes is given by the multi-dimensional vector:

$$
\mathbf{v}(t)=\hat{\mathbf{u}}(t) \quad \mathbf{u}(t)
$$

Each of the dimensions of the vectors, $\mathbf{u}(t)$ and $\hat{\mathbf{u}}(t)$, corresponds to the physical dimension of the sensory input, the motor output or the abstract mathematical dimension in the equations representing the sensory or output signals. For instance, the physical dimension can be light intensity (for vision), sound intensity (for hearing) or chemical intensity (for smelling). It can also be any abstract mathematical dimension (such as pain, which is an abstraction of the alarm signal extracted from the various sensory dimensions to represent a new abstract sensory dimension called "pain," which signifies any potential for tissue damage in biological organisms or the probability for system destruction that endangers an autonomous robot's survival).

If the expected and the actual outcomes are the same, there is no discrepancy between them; the autonomous system is functioning accurately without any errors. This is indicated by $\mathbf{v}(t)=0$. However, if there is a discrepancy between the outcomes, i.e., $\mathbf{v}(t)$ is non-zero $(\mathbf{v}(t) \quad 0)$; then some errors must have existed. Therefore, the disparity vector, $\mathbf{v}(t)$, can be used as the self-discovery error indicator for an autonomous system to detect the existence of error conditions without any external assistance, i.e.,

\section{$\mathbf{v}(t) \quad 0 \quad$ Error condition has discovered}

This multi-dimensional disparity vector is crucial in detecting and identifying the existence of error condition that an autonomous system encountered.

\subsection{Biological Self-Detection of Error using Emotion as the Internal Feedback}

In biological evolution, this disparity vector is evolved as the emotion, which signifies to an animal that something is wrong, i.e., something is incongruent with the expected real world conditions. That is, animals have evolved to use emotion as a guide to detect something went wrong autonomously without needing any external assistance or guidance. Without emotion, an animal is oblivious to the error conditions, and would not be able to respond to the erroneous conditions for subsequent correction. With emotion, animals are able to respond to the fault condition, and do something to correct it.

This also exemplifies traditional robots without emotions cannot respond to any fault conditions autonomously unless the fault conditions and corrective actions were explicitly instructed by some external sources, which is usually beyond the reach (or the ability) of the robot to self-discover and/or self-correct such unexpected/unforeseen errors. 


\subsection{Identification of Source of Errors}

Once the error conditions are self-discovered, the next step in the process of self-correcting error is to identify where the source of errors is coming from. It is crucial to identify the source of error if corrective actions were done to minimize the errors of the system autonomously. In general, there are three possible sources of errors:

(a) input errors (sensory errors),

(b) output errors (execution errors), and

(c) internal modeling errors (prediction errors).

Let $\mathbf{x}$ be the multi-dimensional vector representing the input of the system, $\mathbf{y}$ the vector representing the output of the system, and $\mathbf{z}$ be the vector representing the internal prediction; then the input error, output error and prediction error are represented by $\square \mathbf{x}, \square \mathbf{y}$, and $\square \mathbf{z}$, respectively. Note that any of the above three different sources of errors can contribute to the overall errors that result in producing a discrepancy between the expected and the actual outcomes. Since in the real world, there is usually no a priori knowledge of where the errors may come from, it is one of the most difficult tasks to identify the source of error conditions autonomously. Even though there may not be any clues for identifying the source of error without any $a$ priori knowledge, it is not necessarily insurmountable to figure out the possible source of error. We will introduce the methodologies for detecting and identifying the sources of errors.

\subsection{Identifying the Source of Error}

One of the methodologies to identify the error source is to make an assumption of where the error may come from. Since the errors could come from any one of the above three sources; therefore, by using an exhaustive search method, it can narrow down the source of error one by one. That is, by assuming the source of error is coming from one (or more) of the above sources, then corrective actions can be taken to minimize the encountered errors. Even though this may seem like a brute-force approach to solve the problem; it is, essentially, what evolution had come up with to solve the autonomous error-recovery problem in the emotional resolution process in intellectual higher-animals. This is also one of the reasons why it is often difficult to resolve unresolved emotions (even in humans) when the source of unhappiness is unidentified.

Furthermore, many of the methodologies in artificial intelligence (AI) are essentially using bruteforce approach solutions to solve hard problems without any explicit solutions. These brute-force approaches are generalizable implicit methods using intelligent searches, even without explicit mathematical solutions to solve complex and ill-defined problems. The widely used AI methods for solving these hard problems without explicit solutions include, but not limited to, genetic algorithm [17-19], evolutionary algorithm [2022], artificial neural network [23-29], simulated annealing [30-32], or fuzzy logic approach [33-36]. Each of these search algorithms essentially uses best-guess approaches to search for possible solution, and then uses an iterative approach to minimize the errors in the search in order to arrive at some optimal solutions.

Since numerous search algorithms for exploring possible optimal solutions have already been investigated in these subfields of AI, including genetic algorithm, artificial neural network and simulated annealing, the choice of implementing any of these search methods depends on the implementation of the specific autonomous system. Therefore, the focus of this paper is to explore the methodologies in autonomously detecting and correcting self-discovered errors rather than focusing on selecting which specific corrective algorithm to use, as they had been examined extensively elsewhere in the respective subdisciplines of AI mentioned above.

\subsection{Optimization Process for Discovery and Minimization of Errors}

Toward the goal of identifying the source of unidentified errors in an autonomous system, we introduce the methodology for troubleshooting its own system by the use of the disparity vector, $\mathbf{v}(t)$, as a metric for self-discovering error of the system autonomously. The disparity vector is a time-varying, multidimensional vector that represents the real-time discrepancy between the expected and the actual outcomes in the real world. The existence of error conditions is discovered by the disparity between the expected and the actual outcomes in actuality. Thus, the autonomous process for self-correcting the encountered error is a minimization process to minimize the disparity. The probability of survival of the system is dependent on the accuracy of its predictions. The accuracy of its prediction can be determined by the degree of congruency between the predicted outcome and the actual outcome.

The disparity vector is a measure of the incongruence between the predicted and actual outcomes. Then the goal of the autonomous system is to minimize the disparity vector such that congruence between the prediction and the actuality can be reached. If there is congruence between the predicted and actual outcomes, the errors are minimized, and the probability of survival increases. In other words, this process is essentially an optimization process to minimize the error to obtain an optimal solution for the system to increase its probability of survival. This optimization process is the equivalent of the "survival of the fittest" 
principle in biological evolution by using an iterative search algorithm to eliminate unfit solutions that decrease its survivability.

Thus, the optimization process is an iterative process, through trial-and-error to obtain an optimal solution, similar to the trial-and-error approach to use exhaustive search in genetic algorithm and artificial neural network. Note, it is well known that these AI methods do not necessarily guarantee the solution reaches either a global minimum or a local minimum. They merely provide a solution that minimizes the error conditions because, most often, the global minimum is an unknown in the real world. That is, the most optimal solution may not be known with any a priori knowledge. The best one could do is to find a minimum, which is often a local minimum. Even though it may be a local minimum, it is a solution; regardless of whether it is the most optimal solution or not.

\subsection{Topology of the Solution Space in Search for the Optimal Solution}

Fig. 1 illustrates the topology of the solution space in the error minimization process, in which there may be multiple minima in the multi-dimensional solution space. Since most search algorithms, including artificial neural network, use gradient-descent as one of the feedback criteria in the iterative search for an optimal solution, Fig. 1 provides an illustration of the local minima vs. the global minima in the search space. Using gradient-descent, the search algorithm can often be stuck in a local minimum. Which local minimum it is stuck on often depends on the initial conditions and other search parameters.

Nonetheless, as long as there is a reduction of error, i.e., error minimization, it is correcting itself autonomously without any external guidance. Similarly, in biological evolution, the autonomous solutions found in biological systems are not necessarily the most optimal solution, which is why different animals have evolved with different optimal solutions to similar problems, and came up with different self-corrective strategies. In other words, the search algorithm does not necessarily find a unique solution; most often, it finds different solutions depending on the search path. As long as a solution is found that reduces the error, then the system is able to recover from the self-discovered error autonomously without any external assistance. In real world application, it is often useful to find a practical solution, rather than an absolute solution, in dealing with real world problems. Thus, this paper is focused on deriving the pragmatic solutions to self-discover and self-correct any unexpected/unforeseeable error conditions that an autonomous system has encountered.

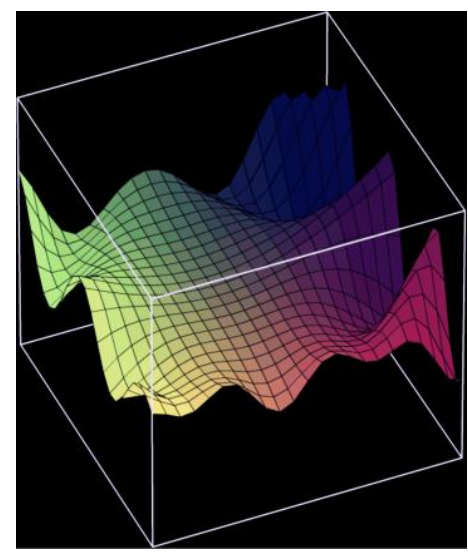

Figure 1. A graphical illustration of the multi-dimensional solution space that most search algorithms use in finding an optimal solution using gradient-descent methods in the optimization process to search for error minima. Note that there can be multiple error minima in the topology. Thus, the optimization process may discover a local minimum rather than a global minimum. Nonetheless, it is an optimal solution, even though it may not be the most optimal solution.

\section{PRIORITIZATION OF ERROR CONDITIONS FOR CORRECTION}

Given that the disparity vector, $\mathbf{v}(t)$, can detect the existence of error conditions, there could be many different error conditions that exist simultaneously in any system. Some of the error conditions may be critical, while others may be less critical. Therefore, it is important to prioritize which error condition needs to be addressed first. In order to prioritize which error condition is more important than others, then the system has to have a mechanism to automate the prioritization process. Most importantly, if all the errors were addressed simultaneously, all the different corrective actions may interfere with each other, resulting in

Self-Corrective Autonomous Systems using Optimization Processes for Detection and ... (Nicoladie Tam) 
a more chaotic system than it was. Thus, it is essential to prioritize the corrective actions in the order of importance, i.e., crucial to the survival of the system as a whole. If the errors are minor, they can be ignored without causing system failure. However, if the errors are major, and if they were ignored, then it would likely lead to catastrophic system failure, decreasing its survival as a system.

\subsection{Prioritization by the Size of Error}

The seemingly logical approach is to prioritize the importance of the error condition is to address the larger disparity first - based on the size of the error it produced (which contributes most to the error conditions). However, in reality, the size of disparity in one dimension cannot be directly compared to the size of another disparity in another dimension in the multi-dimensional space. For instance, using sensory (input) error as an example, errors may exist in both the visual dimension (in vision) and the auditory dimension (in hearing). Therefore, comparing the size of the visual (light) signal with the size of the auditory (sound) signal is meaningless because light and sound signals are completely different from each other in the physical dimension. In other words, the brightness of light cannot be compared to the loudness of sound. Thus, it is not possible to prioritize whether to address the visual disparity or the auditory disparity first based on the size of the error signal itself.

In biological autonomous systems, the prioritization of which error conditions to be addressed first is also a similar issue as in autonomous robots. Most often, the emotional feedback signifies to the animal that something is wrong, yet there may be multiple error conditions that call the attention for the animal to address. Thus, the problem of prioritizing the severity of the error is an important issue to address in autonomous correction of errors. That is, if the autonomous system spends too much time in addressing minor problems to solve instead of correcting the major errors of the system, then it could be stuck in the unresolved state. Furthermore, it can overwhelm the system if every minor error is treated equally because minor errors always exist in the real world; no system is immune to errors.

This is similar to the triage system in emergency care at most hospital's emergency room care system. The prioritization using a triage system will allow the most urgent emergency conditions to be treated first before the less urgent care is performed. This increases the survival of the patients being cared for. By the same token, when the principle of a triage system is used in autonomous recovery of error, it could increase the probability of survival of the system by prioritizing the most urgent error conditions to be addressed first before correcting the minor error conditions.

\subsection{Prioritization by Unexpected Changes}

If it is not possible to prioritize based on the intensity of the error condition due to the different physical dimensions, another methodology is to use the unexpected change as one of the criteria to signify urgency to address the error. That is, rather than comparing the absolute magnitude of error, use the relative error as a measure for comparison. In other words, by using the relative error as a metric identifying the severity of error, it is comparing the same physical dimension based on how the error has progressed whether the error has increased or decreased.

If the error has decreased based on the disparity vector, $\mathbf{v}(t)$, then there is less urgency to address the error. However, if the error has increased, then the priority should be placed higher in the urgency list for addressing. Thus, the rate of change of error would be an appropriate measure to be used as one of the criteria for prioritizing the urgency to address the error conditions.

Using this prioritization principle, the unexpected change in error conditions can be quantified by the rate of change of the disparity vector, $\mathbf{v}(t)$, which is given by the time derivative of the disparity vector,

$\mathbf{v}(t) / t$. If the rate of change of the disparity vector is positive $(\mathbf{v}(t) / t>0)$, it indicates that the error has increased:

$$
\frac{\mathbf{v}(t)}{t}>0 \quad \text { Error has increased }
$$

Using this measure as one of the criteria for prioritizing, then this would indicate urgency in addressing the error condition.

In biological autonomous systems, this urgency to address the error condition is often indicated by the unhappy emotion, which signifies something has gone wrong [14]. Thus, the optimization goal for the animal is to increase the happiness level while decreasing the unhappiness level, in order to reduce the selfdiscovered error condition. This is an autonomic response in animals, which is a built-in mechanism (in-born trait) for animals to response automatically to distress signals. Since the survival of an animal is of utmost importance for an animal, this principle of self-preservation has been evolved as a hardwired feedback 
system in the autonomic nervous system in most higher-animals. Because the autonomic nervous system has been evolved as an automatic self-preservation survival feedback system, most animals are not even aware of such autonomic response consciously. Rather, they respond automatically as an autonomic reflex without reaching the consciousness level, because it is so essential to survival that a rapid response is needed without requiring the complexity of the processing in higher-cognition.

\subsection{Criterion for Indicating Corrective Actions are Effective in Reducing Errors}

Conversely, if the rate of change of the disparity vector is negative $(\mathbf{v}(t) / t<0)$, it indicates that the error has decreased:

$$
\frac{\mathbf{v}(t)}{t}<0 \quad \text { Error has decreased }
$$

This indicates that the corrective actions are effective in reducing the error. Therefore, it can continue the corrective actions until the error reaches a minimum. This would also indicate a lower priority in the urgency list compared to the suddenly discovered errors $\left({ }^{2} \mathbf{v}(t) / t^{2}>0\right.$, see section below), which should be higher in the priority list.

In biological autonomous systems, a reduction of error condition is often indicated by a relief in the unhappy emotion, which serves as an emotional feedback to the animal to relax and not worry so much about the error condition because things are starting to go in the right direction. This also reduces the distress signal emotionally to the animal, so that it does not need to pay as much attention to the error condition as it was before. This often offloads the urgency in the priority list, so that attention can be paid to other more urgent and severe error condition than the one that is starting to show a relief.

\section{CRITERIA FOR DETECTING EFFECTIVE OR INEFFECTIVE CORRECTIVE STRATEGY}

Once the error conditions have been detected and prioritized, then the error correction phase is deployed to correct the encountered error. The system needs to have some form of feedback to indicate whether the corrective actions are proceeding to minimize the errors. Since in deploying best-guess algorithms or exhaustive search strategies in self-correction, there is no guarantee that such corrective actions are effective. Therefore, it is important to have some criteria for detecting whether the deployed corrective actions are effective or not.

In order to quantify the effectiveness of the corrective actions, some criteria are needed to signify to the system whether to continue the previous corrective action or to change to some other alternate corrective actions. One of the criteria for detecting the effectiveness of the corrective actions is the rate of change of the disparity vector $(\mathbf{v}(t) / t)$ because it represents whether the error (disparity) is increasing, decreasing or constant (no change)

\subsection{Criterion for a Change of Corrective Strategy is Needed}

If the rate of change of the disparity vector is zero $(\mathbf{v}(t) / t=0)$, then there is no change in error:

$$
\frac{\mathbf{v}(t)}{t}=0 \quad \text { No change in error }
$$

Therefore, if the corrective actions did not make any changes to the error condition, then it suggests that the corrective actions are ineffective. Thus, it may need to deploy other corrective strategies to correct such an error.

So a zero in the disparity vector $(\mathbf{v}(t) / t=0)$ can be used as the criterion (metric) that indicates the corrective strategy is ineffective. This should send a feedback signal to the system that a change to an alternate corrective action is needed in order to search for some other more effective corrective actions.

\subsection{Criteria for Changing the Direction of Error Correction}

If the corrective actions are proceeding in the correct direction, the errors should reduce. However, if the corrective actions were increasing the errors, then a change of direction would be needed for the corrective actions.

Thus, the rate of change of the disparity vector $(\mathbf{v}(t) / t)$ can also be used to indicate whether the corrective efforts are successful in reducing the error. In other words, whatever the corrective actions are, they may or may not be reducing the errors. Thus, it is important to design an autonomous system that is

Self-Corrective Autonomous Systems using Optimization Processes for Detection and ... (Nicoladie Tam) 
capable of identifying whether its own corrective actions are appropriate or not in minimizing the errors without any external cues. If the corrective actions are maximizing the errors instead of minimizing the errors, then such corrective actions have to be reversed.

Therefore, in designing an intelligent system, an autonomous feedback can be build into the system so as to inform the system whether to continue the corrective actions (to minimize the errors) or reverse the direction of the corrective actions (if the errors is increasing instead of decreasing).

\subsection{Criteria for Reversing the Direction of Error Correction}

The criteria for determining the direction of corrective actions are provided by the rate of change of the disparity vector, i.e., $\mathbf{v}(t) / t$. That is, if the rate of change of the disparity vector is positive (

$\mathbf{v}(t) / t>0$ ), then the error has increased, so the course of corrective actions needs to be reversed in order to minimize the error:

$$
\frac{\mathbf{v}(t)}{t}>0 \quad \text { Reverse the direction of corrective actions }
$$

The direction of the course of action in correcting the error can be reversed by a change in sign of the corrective action mathematically, i.e., multiplied by -1 .

In biological autonomous systems, the increase in the size of error condition is often signified by an increase in the unhappy emotional intensity. The unhappier an animal feels, the more it signifies internally that something is going wrong further and further. The emotional feedback is telling the animal implicitly that it is not going in the right direction to resolve the emotion; something must be done to correct it even though it may be aware of exactly what needs to be done to correct the erroneous corrective actions. Such unhappy (sad) emotion often keeps the animal from continue with the previously attempted solution, which is an attempt to reverse the direction of the corrective actions. It is often stuck in this state of inaction when the animal is experiencing the state of sadness [37].

\subsection{Criteria for Keeping the Same Direction of Corrective Actions}

On the other hand, if the rate of change of the disparity vector is negative $(\mathbf{v}(t) / t<0)$, then the error has decreased. This indicates that the optimization process is proceeding in the correct direction to minimize the error. Therefore, it can stay the same course of action and proceed as usual without changing the direction of the corrective action:

$$
\frac{\mathbf{v}(t)}{t}<0 \quad \text { Stay the course of direction in corrective actions }
$$

This indicates that the corrective actions are effective in reducing the errors.

In biological autonomous systems, this effective corrective action is indicated by the emotional feedback as a state of content or satisfaction that things are going in the right direction. When an animal experiences the feeling of content, there is no need to change any of its courses of action. Therefore, these autonomous criteria are consistent with the principles of operation in animals that had discovered in animal's self-corrective strategies in evolution, even though the animal may not be aware of such strategies consciously. That is because conscious awareness requires cognitive processing to evaluate the effectiveness of such strategy using logical analysis. On the other hand, the emotional responses in the autonomic nervous system of animals often do not reach conscious awareness due to the urgency in the automatic triggering of the hardwired response to address the error conditions for survival. These responses are often evolved as appropriate responses to detect and self-correct the error conditions. They are hardwired as automatic reflexes in animals without needing conscious awareness. The hardwired response decreases the reaction time, so that the animal can address the error conditions as fast as possible for survival. On the other hand, if it reaches conscious level, it would require much longer processing time to assess the situation. It could be too late to respond appropriately if the error conditions are critical to the survival of the animal.

\section{CRITERIA FOR DETECTING WHETHER MINIMA OR MAXIMA HAD REACHED}

Given that the direction of the corrective action has been identified and selected to minimize the errors, then the next step is to identify whether the minima or the maxima had reached. One of the classical 
methods to identifying the maxima and the minima is the use of the second time derivative of the disparity vector, i.e., ${ }^{2} \mathbf{v}(t) / t^{2}$. If a maximum has reached, then ${ }^{2} \mathbf{v}(t) / t^{2}>0$,

$$
\frac{{ }^{2} \mathbf{v}(t)}{t^{2}}>0 \quad \text { Maximum has reached }
$$

However, if a minimum has reached, then ${ }^{2} \mathbf{v}(t) / t^{2}<0$,

$$
\frac{{ }^{2} \mathbf{v}(t)}{t^{2}}<0 \quad \text { Minimum has reached }
$$

Moreover, if a point of inflection has reached, then ${ }^{2} \mathbf{v}(t) / t^{2}=0$,

$$
\frac{{ }^{2} \mathbf{v}(t)}{t^{2}}=0 \quad \text { A point of inflection has reached }
$$

Therefore, using these criteria, the system would be able to identify whether the corrective actions have been successful to minimize the error.

\subsection{Criteria For Stopping Corrective Actions To Prevent Over-Correction}

Most importantly, the system has to be able to stop the corrective actions once the errors has been minimized. Otherwise, the system can over-correct the errors, making the situation worse than before. Therefore, the system has to have a build-in process that indicates whether corrective actions need to be stopped if the errors had reached a minimum.

One of the criteria to stop its own corrective action is when the optimization process has reached its minimum in error reduction. Therefore, the "stop" signal for the system to self-correct itself is the metric indicated by ${ }^{2} \mathbf{v}(t) / t^{2}<0$, which represents a minimum has reached (so that it would not over-correct the errors).

Therefore, the criterion (metric) for determining that a minimum has reached is given by ${ }^{2} \mathbf{v}(t) / t^{2}<0$, i.e.,

$$
\frac{{ }^{2} \mathbf{v}(t)}{t^{2}}<0 \quad \text { Error minimum has reached }
$$

so that the system can stop the corrective actions. Otherwise, it could over-correct it, resulting in unnecessary error conditions. Thus, it is important to detect such a state when it reaches the error minimum to prevent over-correction, which can result in producing oscillatory corrective actions.

In biological autonomous systems, when the animal detects the error had reached a minimum, it is signified by the state of content (happiness) so that it would stop worrying about any errors that it had corrected. Thus, it serves as a signal to tell the animal stop fixing when it has resolved its unhappy emotions. Thus, the state of content feeling often relaxes the animal so the animal would stop trying to make any corrective action, because there is nothing else to fix, and start to enjoy life as it is.

\subsection{Damping of Oscillatory Over-Corrective and Under-Corrective Actions}

Oscillatory behaviors could exhibit if corrective actions are not stopped as soon as the error minimum has been reached. When corrective actions continues after the error minimum has reached, then it will over-correct, and then under-correct subsequently when it detects error starts to increase again after the minimum has reached. Thus, if the error minimum is not detected soon enough, it will produce the classical over-correct and under-correct oscillatory behavior in feedback control.

Furthermore, time delays always exist in a feedback system such that the signal (metric) indicating that the minimization of errors has reached may come too late (due to time delays), some over-corrections of error may be inevitable. Therefore, in order to resolve the oscillatory corrective actions, damping may be needed in the corrective differential equations to reduce the oscillatory corrections:

$$
m \frac{{ }^{2} \mathbf{v}(t)}{t^{2}}+b \frac{\mathbf{v}(t)}{t}+k \mathbf{v}(t)=0, \text { with } m>0, b \quad 0 \text { and } k>0
$$


The above characteristic differential equation has a set of characteristic roots, which is given by:

$$
\frac{b \pm \sqrt{b^{2} 4 m k}}{2 m}
$$

There are three types of damping that may exist, depending on the parameters of the differential equation:

(a) over-damping when $b^{2}>4 m k$;

(b) under-damping when $b^{2}<4 m k$;

(c) critical damping when $b^{2}=4 m k$.

The type of damping is dependent on the sign of the discriminant (i.e., the expression under the square root of the characteristic root) in Eq. (13).

For the over-damping case, the roots are real numbers. For the under-damping case, the roots are imaginary numbers. For the critical damping case, the root is $b / 2 m$. Therefore, ideally, the system can be designed to use critical damping to reduce the oscillatory corrective actions. This is the optimal solution, in which the system is neither over-damped nor under-damped. It will arrive at the error minimum without any oscillation in its corrective actions.

\subsection{Criteria for Alarm for Unexpected Sudden Surprises}

Most importantly, an autonomous system should be able to trigger an alarm if the error conditions are severe. As explained earlier, the severity of the error conditions is difficult to compare because of the differences in physical dimension in the sensory and the motor signals; therefore, the absolute magnitude of the errors is not relevant for comparison. Rather, the relative magnitudes can be used as the criteria for alarm when the errors occurred suddenly.

One of the criteria (metrics) for setting the surprise alarm is the condition in which the error has reached a maximum. When the error is maximized, it should set an alarm condition to signify to the system that some drastic errors have occurred. Since the second time derivative of the disparity, ${ }^{2} \mathbf{v}(t) / t^{2}$, represents how fast the error has changed, it can be used as an alarm signal to indicate that sudden change of errors has occurred. This should trigger urgency for immediate attention to address the error conditions.

Thus, the second time derivative of the disparity vector, ${ }^{2} \mathbf{v}(t) / t^{2}$, can also be used to signal an alarm condition if a maximum has reached, i.e., ${ }^{2} \mathbf{v}(t) / t^{2}>0$,

$$
\frac{{ }^{2} \mathbf{v}}{t^{2}}>0 \quad \text { Set an alarm when maximum error has reached }
$$

\subsection{Biological Response to Emotional Distress to Sudden}

In biological autonomous systems, this alarm is often triggered by a sudden change of error condition, which corresponds to the emotional shock or distress signal as a feedback to the organism to prepare for the worst-case scenario. This is the surprise that animals often get when they realize something drastically went wrong all of a sudden. It prepares the animals to respond to the unexpected emotionally. This state of distress often wakes up animals from the normal state to direct attention to the endangering signals that threaten the survival of the animal.

This emotional shock is an indicator to an animal that signifies a sudden occurrence of event that is not expected. The more sudden it occurred, the more surprise the animal will experience. The emotional trauma is a consequence of the shock that triggered a reaction to deal with the unexpected condition, which the animal is unprepared. The distress condition indicates that something drastically is wrong while no solution has been found yet to resolve the unexpected issues. Without such emotional response to the sudden onset of the distress signal, an animal may not be prepared for the unexpected to happen, even though it is unpredictable how such unforeseen circumstances had occurred. Thus, it is also crucial for autonomous robots to be equipped with such an emotional surprise indicator as one of the criteria to detect unexpected error conditions. 


\section{DISCUSSION}

The theoretical framework for the self-discovery of error conditions and self-correction of the encountered errors are provided in this paper. Using the biologically inspired principles, these methodologies correspond to the strategies and criteria used in biological autonomous systems, including higher-animals and humans. The derivation of the strategies and quantitative criteria are provided with detailed analysis of the rationales behind such methodologies in autonomous control. Using the biologically inspired framework as the principle of operation for autonomous systems, this paper has derived a set of equations to serve as quantitative criteria for the detection of unexpected and unforeseeable error conditions. It has provided a set of automated criteria for triggering self-corrective actions autonomously, similar to the emotional feedback system in response to the self-discovered error conditions in animals.

The use of the disparity measure to quantify the discrepancy between the expected and the actual outcomes in the real world has been validated experimentally in humans. Humans often exhibit the happy emotion that corresponds to the congruence between the expected and the actual outcomes [15]. The decision in producing the corrective actions is also influenced by the emotional feedback, which serves as an internal guidance signal for self-corrective behavior [38].

The optimization of the disparity measure can also be evaluated relative to the cost function with respect to the gains and losses of the predicted outcomes. The model is also consistent with the decision in response to the emotional feedback in correcting the self-discovered disparity between the expected and the actual outcomes, which was examined with the use of fairness to assess the perceptual biases in the sensory input for corrective actions [39]. Furthermore, the model is also consistent with the decision to correct the self-discovered errors, which can be influenced by the inclusion of other autonomous systems [40] in social interactions in terms of empathy, fairness [41] and even jealousy [42]. These principles of social interactions were extended theoretically [43] in autonomous social robots [44-48] and swarm multi-robots [49-54]. Thus, the principles of operation in autonomous control using emotion as the key for internal guidance and feedback correction are validated both experimentally in humans and theoretically by quantitative emotional models that included not just isolated autonomous systems, but also social interacting autonomous systems [55].

Most importantly, the current model of self-correcting autonomous control essentially embodied the ability to acquire emotions implicitly without explicitly programming emotional response into the system.

Last, but not least, the current model not only can exhibit emotions by naturally acquiring the emotional responses, but also can exhibit different personality by the differences in the emotional responses it acquired. Thus, the question whether robots can exhibit personality is not an imagination, but can become a reality based on the current principles of operation in autonomous self-correction of error because personality differences are characterized by the differences in how an organism (or autonomous robot) responds differently according to the environment differently $[56,57]$.

\section{CONCLUSION}

The theoretical derivations of the quantitative methods for self-detection of unexpected errors are provided, which show that the disparity vector can be used to detect any unforeseeable error conditions autonomously without any external assistance. The disparity vector is a time-dependent multi-dimensional vector that represents the discrepancy between the expected outcome predicted by the autonomous system and the actual outcome in the real world. This vector can be used as the metric for self-detection of any encountered errors. The quantitative criteria are also provided for prioritizing the urgency of which corrective actions to proceed first in the optimization process for the minimization of self-discovered errors. The biological correspondences of the emotional feedbacks in autonomous self-detection and self-correction of unexpected errors are also provided to validate the theoretical derivations.

\section{ACKNOWLEDGEMENTS}

The author greatly appreciates Ms. Laura Ouellet for the helpful suggestions and for proofreading the manuscript.

\section{DISCLOSURE}

The author declares that there is no conflict of interest regarding the publication of this paper. 


\section{REFERENCES}

[1] R. A. Chadwick, "Operating multiple semi-autonomous robots: Monitoring, responding, detecting," in Proceedings of the Human Factors and Ergonomics Society Annual Meeting, 2006, pp. 329-333.

[2] W. E. Haisler, J. A. Stricklin, and J. E. Key, "Displacement incrementation in non- linear structural analysis by the self- correcting method," International Journal for Numerical Methods in Engineering, vol. 11, pp. 3-10, 1977.

[3] S. B. Wicker, Error control systems for digital communication and storage vol. 1: Prentice hall Englewood Cliffs, 1995.

[4] R. Shadmehr, M. A. Smith, and J. W. Krakauer, "Error correction, sensory prediction, and adaptation in motor control," Annual review of neuroscience, vol. 33, pp. 89-108, 2010.

[5] C. E. Garcia and M. Morari, "Internal model control. A unifying review and some new results," Industrial \& Engineering Chemistry Process Design and Development, vol. 21, pp. 308-323, 1982.

[6] M. Desmurget and S. Grafton, "Forward modeling allows feedback control for fast reaching movements," Trends in cognitive sciences, vol. 4, pp. 423-431, 2000.

[7] Y. Baryshnikov, E. Coffman, N. Seeman, and T. Yimwadsana, "Self-correcting self-assembly: growth models and the Hammersley process," in DNA Computing, ed: Springer, 2006, pp. 1-11.

[8] C. W. Tseng, W. Lu, and M. P. Baker, "Self-checking and self-correcting internal configuration port circuitry," ed: Google Patents, 2012.

[9] M. F. Angelo, G. D. Wisecup, and D. L. Collins, "System for validating a bios program and memory coupled therewith by using a boot block program having a validation routine," ed: Google Patents, 2003.

[10] H. Wasserman and M. Blum, "Software reliability via run-time result-checking," Journal of the ACM (JACM), vol. 44, pp. 826-849, 1997.

[11] P. Prata and J. G. Silva, "Algorithm based fault tolerance versus result-checking for matrix computations," in Fault-Tolerant Computing, 1999. Digest of Papers. Twenty-Ninth Annual International Symposium on, 1999, pp. 4-11.

[12] D. Tam, "EMOTION-I model: A biologically-based theoretical framework for deriving emotional context of sensation in autonomous control systems," Open Cybern Sys J, vol. 1, pp. 28-46, 2007.

[13] D. Tam, "EMOTION-II model: A theoretical framework for happy emotion as a self-assessment measure indicating the degree-of-fit (congruency) between the expectancy in subjective and objective realities in autonomous control systems," Open Cybern Sys J, vol. 1, pp. 47-60, 2007.

[14] D. N. Tam, "Computation in emotional processing: quantitative confirmation of proportionality hypothesis for angry unhappy emotional intensity to perceived loss," Cogn Comput, vol. 3, pp. 394415, 2011/06/01 2011.

[15] N. D. Tam, "Quantification of happy emotion: Proportionality relationship to gain/loss," Psychol Behav Sci, vol. 3, pp. 60-67, April 6, 20142014.

[16] J. A. Stricklin and W. E. Haisler, "Formulations and solution procedures for nonlinear structural analysis," Computers \& Structures, vol. 7, pp. 125-136, 1977.

[17] H. Mühlenbein and D. Schlierkamp-Voosen, "Predictive Models for the Breeder Genetic Algorithm I. Continuous Parameter Optimization," Evolutionary Computation, vol. 1, pp. 25-49, 1993/03/01 1993.

[18] M. Kumar, M. Husian, N. Upreti, and D. Gupta, "Genetic algorithm: Review and application," International Journal of Information Technology and Knowledge Management, vol. 2, pp. 451-454, 2010.

[19] R. Sivaraj and T. Ravichandran, "A review of selection methods in genetic algorithm," International journal of engineering science and technology, vol. 3, 2011.

[20] C. M. Fonseca and P. J. Fleming, "An overview of evolutionary algorithms in multiobjective optimization," Evolutionary computation, vol. 3, pp. 1-16, 1995.

[21] M. Tomassini, "Parallel and distributed evolutionary algorithms: A review," 1999.

[22] E. Zitzler, L. Thiele, E. Zitzler, E. Zitzler, L. Thiele, and L. Thiele, An evolutionary algorithm for multiobjective optimization: The strength pareto approach vol. 43: Citeseer, 1998.

[23] J. Davis, "An Introduction to Neural Networks," Journal of Cognitive Neuroscience, vol. 8, pp. 383383, 1996/10/01 1996.

[24] S. Haykin, Neural Networks: A Comprehensive Foundation: Prentice Hall PTR, 1994.

[25] R. Hecht-Nielsen, "Theory of the backpropagation neural network," in Neural Networks, 1989. IJCNN., International Joint Conference on, 1989, pp. 593-605 vol.1.

[26] J. J. Hopfield and D. W. Tank, ""Neural" computation of decisions in optimization problems," Biol Cybern, vol. 52, pp. 141-52, 1985.

[27] D. Psaltis, A. Sideris, and A. A. Yamamura, "A multilayered neural network controller," Control Systems Magazine, IEEE, vol. 8, pp. 17-21, 1988. 
[28] D. Tam, "Theoretical Analysis of Cross-Correlation of Time-Series Signals Computed by a TimeDelayed Hebbian Associative Learning Neural Network," The Open Cybernetics \& Systemics Journal, vol. 1, pp. 1-4, 2007.

[29] A. Cochocki and R. Unbehauen, Neural networks for optimization and signal processing: John Wiley \& Sons, Inc., 1993.

[30] S. Kirkpatrick, C. D. Gelatt, and M. P. Vecchi, "Optimization by simulated annealing," science, vol. 220, pp. 671-680, 1983.

[31] P. J. Van Laarhoven and E. H. Aarts, Simulated annealing: theory and applications vol. 37: Springer Science \& Business Media, 1987.

[32] S. Kirkpatrick, "Optimization by simulated annealing: Quantitative studies," Journal of statistical physics, vol. 34, pp. 975-986, 1984.

[33] B. Kosko, "Neural networks and fuzzy systems: a dynamical systems approach to machine intelligence/book and disk," Vol. 1Prentice hall, 1992.

[34] R. Tong, "A control engineering review of fuzzy systems," Automatica, vol. 13, pp. 559-569, 1977.

[35] C. C. Lee, "Fuzzy logic in control systems: fuzzy logic controller. II," Systems, Man and Cybernetics, IEEE Transactions on, vol. 20, pp. 419-435, 1990.

[36] T. J. Ross, Fuzzy logic with engineering applications: John Wiley \& Sons, 2009.

[37] N. D. Tam, "Quantitative assessment of sad emotion," Psychol Behav Sci, vol. 4, pp. 36-43, February $12,20152015$.

[38] [38] N. D. Tam, "Quantification of happy emotion: Dependence on decisions," Psychol Behav Sci, vol. 3, pp. 68-74, April 6, 20142014

[39] N. D. Tam, "Quantification of fairness perception by including other-regarding concerns using a relativistic fairness-equity model," Adv in Soc Sci Research J, vol. 1, pp. 159-169, 2014.

[40] N. D. Tam, "Rational decision-making process choosing fairness over monetary gain as decision criteria," Psychol Behav Sci, vol. 3, pp. 16-23, 2014.

[41] N. D. Tam, "Quantification of fairness bias in relation to decisions using a relativistic fairness-equity model," Adv in Soc Sci Research J, vol. 1, pp. 169-178, 2014.

[42] N. D. Tam and K. M. Smith, "Cognitive computation of jealous emotion," Psychology and Behavioral Sciences, vol. 3, pp. 1-7, Dec. 31, 20142014.

[43] N. D. Tam, "A decision-making phase-space model for fairness assessment," Psychol Behav Sci, vol. 3, pp. 8-15, 2014.

[44] T. Balch and M. Hybinette, "Social potentials for scalable multi-robot formations," in Robotics and Automation, 2000. Proceedings. ICRA'00. IEEE International Conference on, 2000, pp. 73-80.

[45] C. Breazeal and B. Scassellati, "A context-dependent attention system for a social robot," $r n$, vol. 255, p. 3, 1999.

[46] M. P. Michalowski, S. Sabanovic, and R. Simmons, "A spatial model of engagement for a social robot," in Advanced Motion Control, 2006. 9th IEEE International Workshop on, 2006, pp. 762-767.

[47] C. Bartneck and J. Forlizzi, "A design-centred framework for social human-robot interaction," in Proceedings of the 13th IEEE International Workshop on Robot and Human Interactive Communication, 2004, pp. 31-33.

[48] C.-Y. Chen and P.-H. Huang, "RETRACTED: Review of an autonomous humanoid robot and its mechanical control," Journal of Vibration and Control, vol. 18, pp. 973-982, 2012.

[49] G. Bilbeisi, N. Al-Madi, and F. Awad, "PSO-AG: A Multi-Robot Path Planning and obstacle avoidance algorithm," in Applied Electrical Engineering and Computing Technologies (AEECT), 2015 IEEE Jordan Conference on, 2015, pp. 1-6.

[50] D. Fox, W. Burgard, H. Kruppa, and S. Thrun, "A probabilistic approach to collaborative multi-robot localization," Autonomous robots, vol. 8, pp. 325-344, 2000.

[51] M. Brambilla, E. Ferrante, M. Birattari, and M. Dorigo, "Swarm robotics: a review from the swarm engineering perspective," Swarm Intelligence, vol. 7, pp. 1-41, 2013.

[52] K. Lerman, A. Martinoli, and A. Galstyan, "A review of probabilistic macroscopic models for swarm robotic systems," in Swarm robotics, ed: Springer, 2005, pp. 143-152.

[53] L. Bayindir and E. Sahin, "A review of studies in swarm robotics," Turkish Journal of Electrical Engineering, vol. 15, pp. 115-147, 2007.

[54] Y. Mohan and S. Ponnambalam, "An extensive review of research in swarm robotics," in Nature \& Biologically Inspired Computing, 2009. NaBIC 2009. World Congress on, 2009, pp. 140-145.

[55] N. D. Tam, "EMOTION-III model: A theoretical framework for social empathic emotions in autonomous control systems," Open Cybern Sys J, vol. in press, 2016. 
[56] K. M. Lee, W. Peng, S. A. Jin, and C. Yan, "Can robots manifest personality?: An empirical test of personality recognition, social responses, and social presence in human-robot interaction," Journal of communication, vol. 56, pp. 754-772, 2006.

[57] K. M. Lee, Y. Jung, J. Kim, and S. R. Kim, "Are physically embodied social agents better than disembodied social agents?: The effects of physical embodiment, tactile interaction, and people's loneliness in human-robot interaction," International Journal of Human-Computer Studies, vol. 64, pp. 962-973, 2006. 